\title{
Aggregation Behaviour and Interspecific Responses in Rhodnius prolixus Stål
}

\author{
Alicia N Lorenzo Figueiras ${ }^{+}$, Claudio R Lazzari \\ Departamento de Ciencias Biológicas, Facultad de Ciencias Exactas y Naturales, Universidad de Buenos Aires, Ciudad \\ Universitaria, Casilla Postal C1428EHA, Buenos Aires, Argentina
}

\begin{abstract}
The response to intra- and interspecific faecal assembling signals was tested in Rhodnius prolixus. Papers impregnated with excrement of $\mathrm{R}$. prolixus induced the aggregation of larvae of this species, but also of those of Triatoma infestans. However, faeces belonging to T. infestans were not able to assemble larvae of R. prolixus. On the other hand, there was no response of R. prolixus to putative chemical factors from their cuticle (footprints), in contrast to $\mathrm{T}$. infestans. Results are discussed as related to the ecology of both species.
\end{abstract}

Key words: aggregation behaviour - faeces - footprints - interspecific communication

Rhodnius prolixus is an haematophagous bug distributed over Venezuela, Colombia, and it is also important in some regions of Central America, specially Guatemala, Honduras and El Salvador. In Venezuela and Colombia, $R$. prolixus inhabits mainly wild environments, such as the tops of palm trees. In Central America, these bugs are adapted to domestic environments (Schofield 1994). However, these reports of $R$. prolixus in sylvatic habitats, especially in palm trees, might be misidentifications of morphologically similar species such as $R$. robustus in Venezuela and Northern Brazil, or R. pallescens in Colombia. In Central America, R. prolixus is adapted to domestic environments. No sylvatic populations of these bugs have been found in Central America, and the status of "sylvatic" $R$. prolixus in Venezuela remains uncertain (Anonymous 2000).

Aggregation behaviour, as a response to chemical signals, has been reported in several species of Triatominae (Velázquez Antich 1968, Baldwin et al. 1971, Schofield \& Patterson 1977, Ondarza et al. 1986, Cruz-López et al. 1993, Lorenzo Figueiras et al. 1994). Schofield and Patterson (1977) have reported an assembly pheromone in the larval faeces of $R$. prolixus that attracts unfed larvae and arrests the locomotion of fed larvae.

In addition to dry faeces, a chemical mark left behind by bugs on walked substrates (footprints) also promotes aggregation in T. infestans (Lorenzo Figueiras \& Lazzari 1998a). It has been proposed that this species present a chemical substance in its cuticle that impregnates surfaces and provokes assembling in the bugs. This signal constitutes an assembling mark acting as a "chemical footprint".

This investigation received financial support from the UNDP/ World Bank/WHO Special Program for Research and Training in Tropical Diseases, CONICET and the Universidad de Buenos Aires, Argentina.

${ }^{+}$Corresponding author. Fax: +54-1-5763384. E-mail: aliciaf@bg.fcen.uba.ar

Received 12 June 2001

Accepted 17 December 2001
The specificity of the faeces signals has been studied by Cruz-López et al. (1993), who reported that an interspecific aggregation signals are present in the excrement of larvae and adults of five species of the subfamily Triatominae (T. mazzotti, T. longipennis, T. pallidipennis, T. barberi and R. prolixus). Furthermore, Lorenzo Figueiras and Lazzari (1998b) showed that an aggregating substance is present in the dry faeces of $T$. infestans, $T$. sordida and $T$. guasayana, which acts both, intra- and interspecifically.

The chemical cues that induce assembling in triatomines have been repeatedly signalled as potential tools for controlling triatomines. In addition, their study becomes relevant to understand the relationship between different triatomine species with their habitat.

The present work was undertaken to examine the response of $R$. prolixus to aggregation substances present in their faeces and to compounds left on papers by means of physical contact (footprints). Furthermore, we analyzed the cross-response of both $R$. prolixus and $T$. infestans to their aggregation signals.

\section{MATERIALS AND METHODS}

The experiments were conducted with 3rd instar larvae of $R$. prolixus and 4th instar larvae of $T$. infestans. All the experimental insects were used ten days after the ecdysis.

To test the response of the bugs to the faeces, these were collected on pieces of filter paper $(3 \times 2 \mathrm{~cm})$ during four days after feeding, avoiding any physical contact with the insect's body. The faeces collected were used $24 \mathrm{~h}$ later. To analyse the response of the bugs to their potential footprints, a group of 20 unfed larvae that had their anus sealed with wax, were placed in a plastic container with pieces of filter paper $(3 \times 2 \mathrm{~cm})$ inside and allowed to walk freely. After ten days, these papers were examined to confirm the absence of faecal spots and used for the experiments.

The experimental device employed to test both kinds of cues consisted of a circular glass arena $13 \mathrm{~cm}$ in diameter divided in three equal virtual sectors (Lorenzo Figueiras et al. 1994). The assays were carried out using a piece of filter paper $(3 \times 2 \mathrm{~cm})$ placed on each sector. One 
paper was contaminated with the corresponding source of stimuli, while the other two remained clean as controls. A group of larvae was carefully released in the centre of the arena and the position of the bugs was recorded after $1 \mathrm{~h}$, by counting the numbers of insects on each sector.

The response to intra- and interspecific faeces was tested. The aggregation behaviour of $R$. prolixus to its own faeces and to the excrements of $T$. infestans was recorded. We performed eight replicates with 8 to 12 insects per assay. In addition, the assembly response of $T$. infestans to faeces of $R$. prolixus was analyzed in six replications, using nine insects per assay.

The aggregation behaviour of $R$. prolixus to its footprints was studied performing two series of assays, differing in the fasting period of the experimental insects. Bugs were fed during the previous instar and used for assays either 6 or 20 days after ecdysis.

For each experimental series the distribution of the insects in the arena was statistically analyzed by means of a G-test for goodness of fit to a random distribution (i.e., $1 / 3$ for the experimental sector and $2 / 3$ for control sectors). The aggregation response was compared using an unpaired t-test.

\section{RESULTS}

Fig. 1 depicts the aggregation responses of $R$. prolixus to its own faeces as well as that to excrements belonging to T. infestans. As previously shown for this species and for T. infestans (Schofield \& Patterson 1977, Lorenzo Figueiras et al. 1994), R. prolixus significantly aggregated around papers impregnated with homospecific dry excrement (G-test, $P<0.001$ ). Furthermore, a significant assembling behaviour was observed when we tested the response of larvae of $T$. infestans to faeces of $R$. prolixus (Fig. 2, G-test, $P<0.001$ ). However, faeces belonging to $T$. infestans were not able to assemble larvae of $R$. prolixus (Fig. 1, G-test, NS).

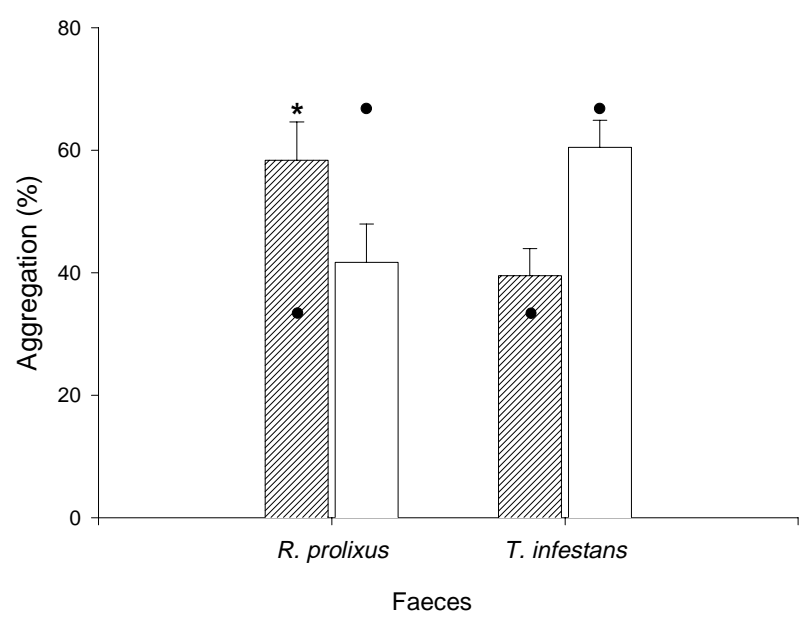

Fig. 1: aggregation response of Rhodnius prolixus to its own faeces and to faeces belonging to Triatoma infestans. The asterisk indicates statistical significance: only the faeces of $R$. prolixus were able to assemble conspecific larvae $(P<0.001)$, but not the excrement of $T$. infestans. The black circles indicate the value expected from a random distribution, i.e., $33 \%$ for the experimental sector (hatched bars) and $66.7 \%$ for the control one (empty bars).
The aggregation level induced in the two named species by faeces belonging to $R$. prolixus was significantly different (t-test; $\mathrm{t}=2.78 ; \mathrm{df}=11 ; P=0.0179 ; 68.7 \%$ in $T$. infestans and $58.3 \%$ in $R$. prolixus). Remarkably, $T$. infestans showed a stronger response than $R$. prolixus to the excrements of the later. Indeed, T. infestans showed a tendency to aggregate around papers impregnated with faeces of $R$. prolixus, similar to that reported for their own faeces (t-test; $\mathrm{t}=1.286 ; \mathrm{df}=15 ; P=0.2178$, NS, Lorenzo Figueiras et al. 1994).

A random distribution was observed when we tested the larvae of $R$. prolixus to its supposed footprints (Gtest, NS, $P>0.05$ ), independently of the bugs' nutritional stage.

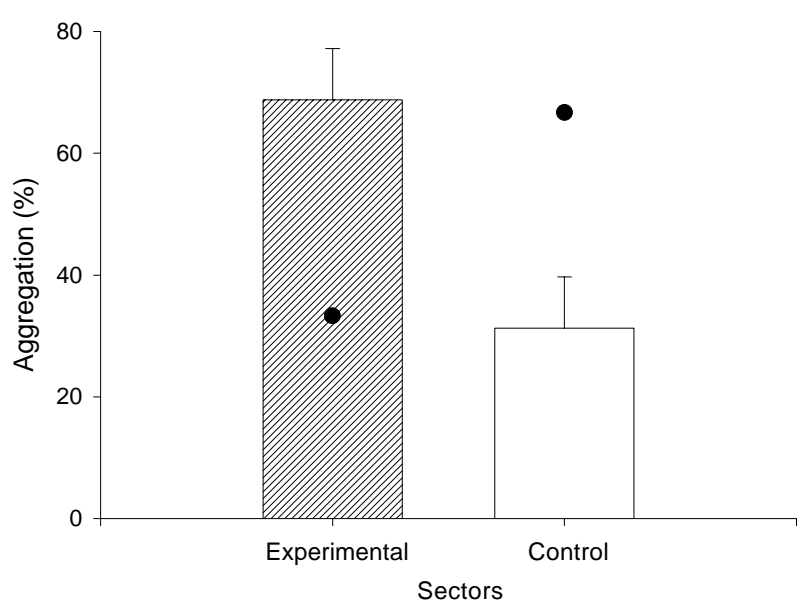

Fig. 2: the significant assembly response of Triatoma infestans to faeces of Rhodnius prolixus. The black circles indicate values expected from a random distribution, i.e., 33\% for the experimental sector and $67 \%$ for the control one.

\section{DISCUSSION}

The results presented here extend our knowledge about the assembling behaviour of triatomines bugs. We confirmed that an assembling substance is present in the dry faeces of $R$. prolixus, which also acts in an interspecific fashion with $T$. infestans. Remarkably, faeces of $R$. prolixus induce assembling of $T$. infestans, but the inverse is not true. This difference between the species could be related with their natural history. $R$. prolixus lives in palm trees, associated to avian nests, and the use of this signal could be less important in the orientation to refuges, than in T. infestans. The fact that larvae of $R$. prolixus did not assemble on faeces of $T$. infestans agrees with a weaker response of that species even to their own faeces, when compared with other triatomines, such as $T$. infestans. Besides, the aggregation response of the two species to faeces belonging to $R$. prolixus was significantly different; $T$. infestans evincing a stronger response than $R$. prolixus.

To comprehend the reasons for this differential response, we can speculate about the different levels on which the response can be modulated. On the one hand, the chemical composition of faeces determines their at- 
tractiveness. On the other hand, the sensory sensitivity of the insect can make it more or less prone to respond. Both factors can be modulated independently by natural selection, as variables related to the adaptation to their particular environments. The interaction here described seems to agree more with the second possibility, i.e. differences in sensitivity, than with the first one. Whereas $T$. infestans would be a species that detects and makes use of faecal signals to find a shelter, independently of their origin (intra- or interspecific), $R$. prolixus exhibits a low tendency to respond, even when excrement belongs to conspecifics. Furthermore, R. prolixus also fails to response to another assembling cue, i.e., cuticular footprints (Lorenzo Figueiras \& Lazzari 1998a), whose presence has been also confirmed in other triatomines (Pires et al. 1999, Vitta et al. 1999). Looking at the habitats of both species, a clear difference appears in the spatial relation between refuges and hosts. In domestic habitats, typically inhabited by $T$. infestans, potential shelters are present in walls and ceilings, i.e., bugs should displace relatively afar to contact a host and to return to a protected place. In this scenario, distant guiding cues are relevant, not only to approach a host, but also to find the return path to a refuge. In the case of $R$. prolixus, bird nests and palm trees offer protection relatively close to the food source. So, this species could simply use the same host cues that guided the approach to it, for moving away of the host to reach a shelter, by inverting the sign of orientation (e.g., negative instead positive thermotaxis or chemotaxis).

Recently, the entity of wild and domestic Rhodnius strains has been revised by Soares et al. (1999). These studies indicate that colonies could be contaminated by different Rhodnius strains. Nevertheless, the same conjectures about the relationship between chemical communication and habitat can be applied to ancient non-domiciliary Rhodnius. Although some of them moved towards human housings and originated the present arrange of species and strains of the genus, the assembling mechanisms could have been conserved if it evolved earlier than the process of domiciliation.

Cruz-López et al. (1993) reported the existence of an interspecific assembling pheromone in the faeces of larvae and adults of T. mazzottii, T. longipennis, $T$. pallidipennis, $T$. barberi and $R$. prolixus. They showed that the faeces of the five species did not exhibit differences in attractiveness for the insects. These authors also noted that faeces belonging to $R$. prolixus induced the weakest assembling response.

These results add new information about the interspecific aggregation behaviour of $T$. infestans that we started to study with other two species: T. sordida and $T$. guasayana (Lorenzo Figueiras \& Lazzari 1998b). The excrement of the last two species induces aggregation in larvae of $T$. infestans. Again, the fact that the chemical factor present in the faeces acts in an interspecific fashion is relevant in two senses. On the one hand, because the individuals of T. infestans could find and exploit refuges used by conspecifics as well as by members of other species inhabiting the same area (e.g. T. sordida and $T$. guasayana, but not $R$. prolixus). On the other hand, because along their evolution triatomines either did not need or have not been able to develop discrete communicating signals of species-specific action. This fact could represent an advantage in the use of assembling cues as baits in capture traps or artificial refuges, since they would be effective for more than one triatomine species, facilitating their use.

\section{ACKNOWLEDGEMENTS}

To the staff members of the Laboratory of Insect Physiology for many fruitful discussions and for correcting the manuscript.

\section{REFERENCES}

Anonymous 2000. Tribe Rhodniini. http://eclat.fcien.edu.uy/ ingles/tribe/l.rhodniini.htm

Baldwin WF, Knight AG, Lynn KR 1971. A sex pheromone in Rhodnius prolixus (Hemiptera: Reduviidae). Can Entomol 103: 18-22.

Cruz-López L, Malo EA, Rojas JC 1993. Aggregation pheromone in five species of Triatominae (Hemiptera: Reduviidae). Mem Inst Oswaldo Cruz 88: 535-539.

Lorenzo Figueiras AN, Lazzari CR 1998a. Aggregation in the haematophagous bug Triatoma infestans: a novel assembling factor. Physiol Entomol 23: 33-37.

Lorenzo Figueiras AN, Lazzari CR 1998b. Aggregation behaviour and interspecific responses in three species of Triatominae. Mem Inst Oswaldo Cruz 93: 133-137.

Lorenzo Figueiras AN, Kenigsten A, Lazzari CR 1994. Aggregation in haematophagous bug Triatoma infestans: chemical signals and temporal pattern. J Insect Physiol 40:312-316.

Ondarza RN, Gutiérrez-Martínez A, Malo EA 1986. Evidence for the presence of sex and aggregation pheromones from Triatoma mazzottii (Hemiptera: Reduviidae). J Econ Entomol 79: 688-692.

Pires HH, Lorenzo M, Diotauiti L, Lazzari C, Lorenzo Figueiras AN 1999. Agregação em larvas de Panstrongylus megistus através de substâncias químicas presentes em fezes e "bodyprints". XVI Congresso de Parasitologia, Brasil.

Schofield CJ 1994. Triatominae. Biología y Control, Eurocommunica Publications, UK, $76 \mathrm{pp}$.

Schofield CJ, Patterson JW 1977. Assembly pheromone of Triatoma infestans and Rhodnius prolixus nymphs (Hemiptera: Reduviidae). J Med Entomol 13: 727-734.

Soares RPP, Barbosa SE, Dujardin JP, Schofield CJ, Siqueira AM, Diotauiti L 1999. Characterization of Rhodnius neglectus from two regions of Brazil using isoenzymes, genitalia morphology and morphometry. Mem Inst Oswaldo Cruz 94: 161-166.

Velázquez Antich A 1968. Atracción por olor en ninfas y adultos de Rhodnius prolixus. Rev Inst Med Trop Parasitol 10: 242-246.

Vitta A, Lorenzo Figueiras AN, Diotauiti L, Lazzari C, Lorenzo M 1999. Agregação mediada por sinais químicos presentes nas fezes e na cutícula de Triatoma pseudomaculata. XVI Congresso de Parasitologia, Brasil. 